\title{
Formation of bipolar planetary nebula M 2-9 by confining toroidal magnetic field and surrounding high-ram pressure wind
}

\author{
Haruichi Washimi ${ }^{1}$, Gary P. Zank ${ }^{1}$, Takashi Tanaka ${ }^{2}$, \\ and Bruce Balick ${ }^{3}$ \\ ${ }^{1}$ Institute of Geophysics and Planetary Physics, University of California, Riverside, \\ CA 92521, USA \\ email:washimih@ucr.edu \\ ${ }^{2}$ Faculty of Science, Kyushu University, Hakozaki, Fukuoka 812-8581, Japan \\ ${ }^{3}$ Department of Astronomy, University of Washington, Seattle, Washington 98195, USA
}

\begin{abstract}
We model the highly axisymmetric planetary nebula M2-9 using a global 3-D MHD simulation. We assume that there is no high-density circumstellar gas around M2-9, because termination shocks are missing at the top of the bipolar lobes in optical images. The overall structure of our MHD solution is a self-consistent consequence of the stellar wind itself, corresponding to two kinds of circumstellar gas distributions, one a collimated flow and the other enveloping the collimated flow. Thus our model shows a 'solitary stellar wind' forming in a diluted circumstellar gas density.
\end{abstract}

Keywords. Planetary nebulae: individual (M2-9), MHD

We model the highly axisymmetric planetary nebula M2-9 using a global 3-D MHD simulation. In this study we assume that there is no high-density circumstellar gas around M2-9, because termination shocks are missing at the top of the bipolar lobes in optical images. Under such conditions, an alternative candidate to collimating lobes may be a toroidal magnetic field. However, because the magnetic field is determined selfconsistently by MHD processes, the magnetic field will expand laterally if there is no confinement effect around the magnetic-field region. Hence, there should be something to prevent the divergence of the magnetic field in the lateral direction, but it cannot be high-density interstellar gas. We performed a numerical simulation based on the ideal time-dependent MHD equations to clarify the formation mechanism of the bipolar lobes.

We find that the bipolar lobes are collimated by the ambient toroidal field which is carried out by the M2-9 stellar wind. The toroidal field is also confined by the surrounding high-speed and low-density stellar wind which originates at lower latitudes of the star and is accelerated outwards by a strong magnetic pressure. The inner and outer boundary conditions of our simulations are the following: a radial wind speed $V=50 \mathrm{~km} / \mathrm{s}$, density $N=10^{6} \mathrm{~cm}^{-3}$, temperature $T=10^{4} \mathrm{~K}$, toroidal field $B_{\phi}=B_{\phi 0} \sin \theta$, and, on the outer boundary sphere, a uniform interstellar medium is prescribed on the upstream side.

For the weak magnetic pressure case $(\alpha=20$, where $\alpha$ is the ratio of the rampressure to the magnetic-pressure), the density is nearly spherically distributed with a weak elongation in the $\mathrm{z}$ direction. The toroidal magnetic field $B_{\phi}$ maintains its initial $\sin \theta$-dependence, and the stellar wind maintains its initial $50 \mathrm{~km} / \mathrm{s}$ velocity $V$ throughout, which means that no acceleration occurs. The temperature decreases adiabatically with distance from the star, and possesses a weak anisotropy associated with the weak magnetic pressure. 
For the case when $\alpha=1.0$, i.e., for the case when the magnetic-pressure is the same as the ram-pressure on the inner boundary, a collimated high-density region is formed in the polar region, and it extends along the polar axis. The magnetic intensity shows an evident enhancement at high latitudes around the collimated flow. The wind at lower and middle latitudes is found to be accelerated to a high-speed of $100 \mathrm{~km} / \mathrm{s}$ or more while the wind along the polar axis maintains its initial speed of $50 \mathrm{~km} / \mathrm{s}$ up to about $20^{\prime \prime}$ extent in both northern and southern directions. Together with the magnetic-intensity pattern, it is obvious that magnetic pressure accelerates the wind in the radial direction at lower and middle latitudes. The ram pressure increases at lower and middle latitudes. The enhanced ram-pressure and strong azimuthal gradient in magnetic pressure indicate that the stellar wind is pushed toward the pole. Hence, the stellar wind is now inclined towards the poles with distance from the star. The temperature is high at higher latitudes, (the result of the adiabatic compression of the wind in the azimuthal direction) and low at middle and lower latitudes (due to adiabatic expansion of the wind). The region of high temperature, at about $65^{\circ}$ latitude and beyond, corresponds to the angle at which the magnetic field is enhanced. Evidently, the enhanced magnetic field spiraling around the pole and the enhanced ram pressure of the stellar wind confine the wind at high latitudes, resulting in the formation of a collimated high-density flow along the polar axis $(\alpha=0.3)$.

Our simulations show that the initial $50 \mathrm{~km} / \mathrm{s}$ wind on the inner boundary is strongly accelerated radially at middle and lower latitudes, attaining a speed of $158-200 \mathrm{~km} / \mathrm{s}$. This compares well to the observed M2-9 polar outflow of $46 \mathrm{~km} / \mathrm{s}$ (Solf (2000)) at the inner region, changing to $164 \mathrm{~km} / \mathrm{s}$ (Schwarz, Aspin, Corradi, \& Reipurth (1997)) at the top of the lobes. The temperature is high at higher latitudes ( $65^{\circ}$ and beyond), and low at middle and lower latitudes, as in the case when $\alpha=1$. The double-shell structure shows that the inner lobes are hot, $10^{4} \mathrm{~K}$, while the outer lobes are cool, $10^{3} \mathrm{~K}$ or less. Accordingly, we identify the observed inner lobes with the high-temperature region of our model simulation, i.e., the region greater than $65^{\circ}$ in latitude, while the surrounding high-density region corresponds to the outer lobes. To conclude, our simulations can explain the basic structure of M2-9.

Although our result shows a collimated structure of the bipolar lobes, the observed density enhancement at the surface of the lobes is not found. However, because the lobes are found to be pushed up from lower to higher latitudes and to be compressed by the accelerated stellar wind, we can expect the density enhancement at the surface will appear in more sophisticated simulations (for example using finer meshes or putting some additional conditions such as a massive or high-speed polar wind (Tanaka \& Washimi $(2002)))$, to be performed in the future.

Numerical computations were carried out with the SX-6 at the NICT in Japan.

\section{References}

Solf, J. 2000, AA 354, 674

Schwarz, H.E., Aspin, R.L.M., \& Corradi, R.L.M., Reipurth, Bo 1997, AA 319, 267

Tanaka, T. \& Washimi, H. 2002, Science 296, 321 